\title{
Automatic building detection and land-use classification in urban areas using multispectral high-spatial resolution imagery and LiDAR data
}

\author{
Txomin Hermosilla \\ Integrated Remote Sensing Studio, Department of Forest Resources Management, University of British Columbia, 2424 \\ Main Mall, Vancouver, BC V6T 1Z4, Canada. \\ Advisor/s: Luis A. Ruiz \\ Date and location of the PhD thesis defense: 8 Julyy 2011, Universitat Politècnica de València
}

Received 8th Jan 2014; accepted 25th May 2014

\section{Abstract}

Urban areas are important environments, accounting for approximately half the population of the world. Cities attract residents partly because they offer ample opportunities for development, which often results in urban sprawl and its complex environmental implications. It is therefore necessary to develop technologies and methodologies that permit monitoring the effects of various problems that have been or are thought to be associated with urban sprawl. These technologies would facilitate the adoption of policies seeking to minimize the negative effects of urban sprawl. Solutions require a precise knowledge of the urban environment under consideration to enable the development of more efficient urban zoning plans. The high dynamism of urban areas produces seemingly continuous alterations of land cover and use; consequently, cartographic information becomes quickly and is oftentimes outdated. Hence, the availability of detailed and up-to-date cartographic and geographic information is imperative for an adequate management and planning of urban areas. Usually the process of creating land-use/land-cover maps of urban areas involves field visits and classical photo-interpretation techniques employing aerial imagery. These methodologies are expensive, time consuming, and also subjective. Digital image processing techniques help reduce the volume of information that needs to be manually interpreted.

The aim of this study is to establish a methodology to automatically detect buildings and to automatically classify land use in urban environments using multispectral high-spatial resolution imagery and LiDAR data. These data were acquired in the framework of the Spanish National Plan for Airborne Orthophotographs, having been available for public Spanish administrations.

Two main approaches for automatic building detection and localization using high spatial resolution imagery and LiDAR data are evaluated The thresholding-based approach is founded on the establishment of two threshold values: one is the minimum height to be considered as a building, defined using the LiDAR data; the other is the presence of vegetation, defined with the spectral response. The other approach follows the standard scheme of object-based image classification: segmentation, feature extraction and selection, and classification, here performed using decision trees. In addition, the effect of including contextual relations

Correspondence to: txomin.hermosilla@live.forestry.ubc.ca

Recommended for acceptance by $<$ Alicia Fornés and Volkmar Frinken $>$

ELCVIA ISSN: 1577-5097

Published by Computer Vision Center / Universitat Autonoma de Barcelona, Barcelona, Spain 
with shadows in the building detection process is evaluated. Quality assessment is performed at both area and object levels. Area-level assessments evaluate the building delineation performance whereas object-level assessments evaluate the accuracy in the spatial location of individual buildings.

Urban land-use classification is achieved by applying object-based image analysis techniques. Objects are defined using the boundaries of cadastral plots. The plots were characterized to achieve the classification by employing a descriptive feature set specifically designed to describe urban environments. The proposed descriptive features aim to emulate human cognition by numerically quantifying the properties of the image elements and so enable each to be distinguishable. These features describe each plot as a single entity based on several aspects that reflect the information used: spectral, three-dimensional, and geometric typologies. In addition, a set of contextual features at both the internal and external levels is defined. Internal context features describe an object with respect to the land cover types contained within the plots, which were, in this case, buildings and vegetation. External context features characterise each object by considering the common properties of adjacent objects that, when combined, create an aggregate in a higher level than plot level: urban blocks.

Results show that thresholding-based building detection approach performs better in the different scenarios assessed. This method produces a more accurate building delineation and object detection than the object-based classification method. The building type appears as a key factor in the building detection performance. Thus, urban and industrial areas show better accuracies in detection metrics than suburban areas, due to the small size of suburban constructions, combined with the prominent presence of trees in suburban classes, hindering the building detection process. The relations between buildings and shadows improve the object-level detection, removing small objects erroneously detected as buildings that negatively affect to the quality indices.

Classification test results show that internal and external context features complement the image-derived features, improving the classification accuracy values of urban classes, especially between classes that show similarities in their image-based and three-dimensional features. Context features enable a superior discrimination of suburban building typologies, of planned urban areas and historical areas, and also of planned urban areas and isolated buildings.
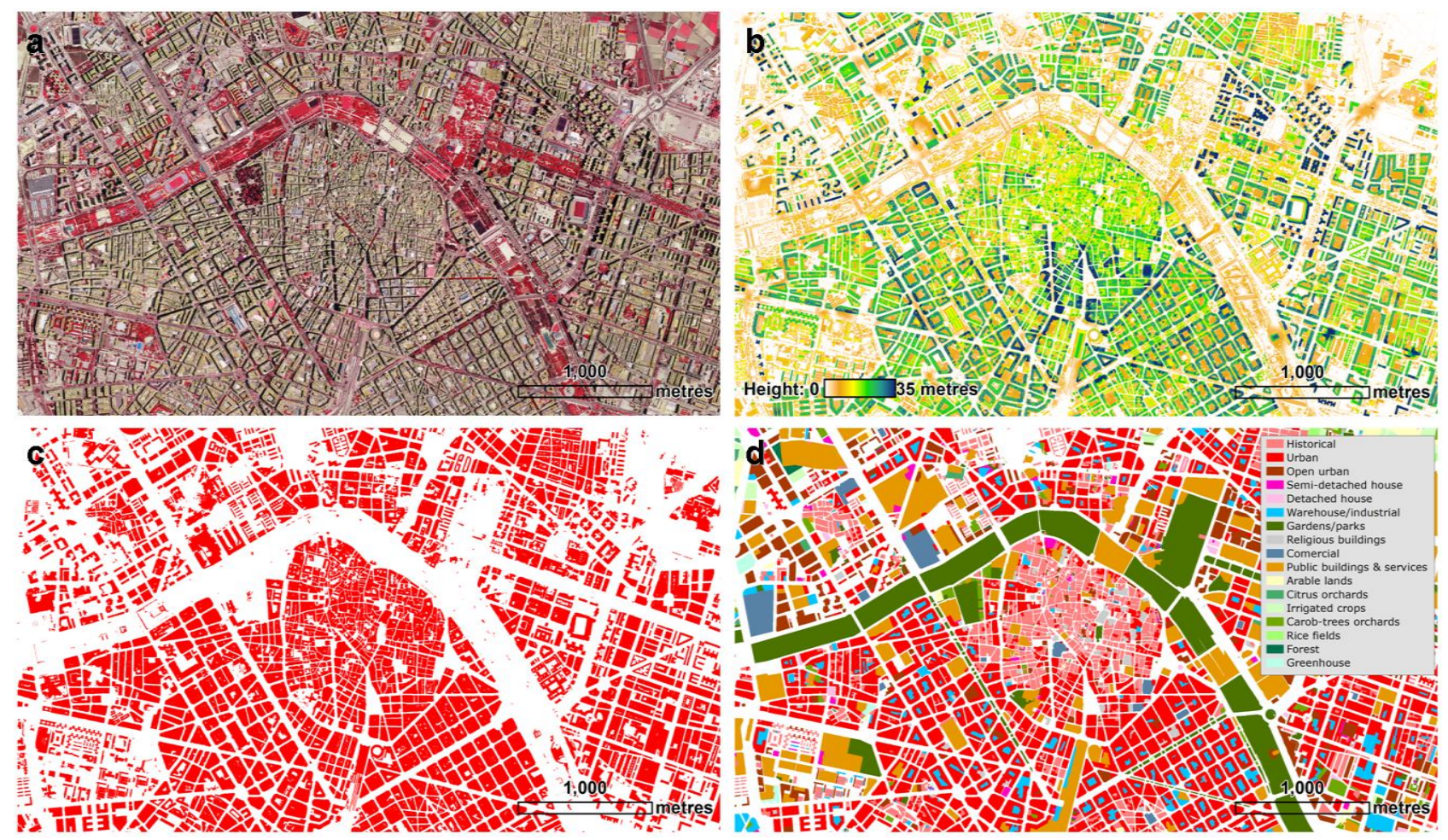

Figure 1: (a) Colour infrarred composition of the urban centre of Valencia (Spain), (b) normalised digital surface model created with the LiDAR data, (c) result of the automatic building detection process; and (d) thematic map composition showing the land use classes assigned to each cadastral plot. 
The outcomes show that these automatic methodologies are especially suitable for computing useful information for constructing and updating land-use/land-cover geospatial databases. Digital image processing-based methodologies provide better results than visual interpretation-based methods. Thus, automatic building detection techniques produce a superior estimation of built-up surface in an objective manner, independent of human operators. The combination of building detection and automatic classification of land use in urban areas enable the distinguishing and describing of different urban typologies, contributing to greater accuracy and information than standard visual interpretation-based techniques. The proposed methodology, based on an automated descriptive feature extraction from LiDAR images and data, is appropriate for city mapping, urban landscape characterisation and management, and the updating of geospatial databases, all of which provide novel tools to increase the frequency and efficiency of the study of complex urban areas.

\section{References}

[1] T. Hermosilla. "Detección automática de edificios y clasificación de usos del suelo en entornos urbanos con imágenes de alta resolución y datos LiDAR". PhD Thesis. Universitat Politècnica de València, Spain, 2011.

[2] T. Hermosilla, L.A. Ruiz, J.A. Recio, J. Estornell J., 2011. "Evaluation of automatic building detection approaches combining high resolution images and LiDAR data". Remote Sensing, 3(6):1188-1210, 2011.

[3] T. Hermosilla, L.A. Ruiz, J.A. Recio, M. Cambra-López. "Assessing contextual descriptive features for plot-based classification of urban areas". Landscape and Urban Planning, 106(1):124-137, 2012.

[4] T. Hermosilla, J.L. Gil-Yepes, J.A. Recio, L.A. Ruiz. "Change detection in periurban areas based on contextual classification”. Photogrammetrie Fernerkundung Geoinformation, 2012(4):355-366, 2012.

[5] T. Hermosilla, J. Palomar-Vázquez, A. Balaguer-Beser, J. Balsa-Barreiro, L.A. Ruiz. 2014. "Using street based metrics to characterize urban typologies". Computers, Environment and Urban Systems, 44:68-79, 2014. 